\title{
MEDICIÓN DE LA CALIDAD DEL SERVICIO TELEFÓNICO, UTILIZANDO MUESTREO PROBABILÍSTICO
}

\author{
Julio Ramos Ramírez ${ }^{1}$ y Nelly Pillhuamán Caña ${ }^{2}$
}

RESUMEN.- El presente artículo trata sobre la metodología estadística implementada para la supervisión de la instalación de líneas telefónicas, mediante el cálculo de un parámetro establecido dentro de los procedimientos de supervisión, que mide el grado de cumplimiento del servicio brindado por la empresa concesionaria.

PALABRAS CLAVE.- Muestreo probabilístico. Parácimetros de medición de Supervisión.

\section{MENSURATION OF THE QUALITY OF THE PHONE SERVICE, USING PROBABILISTIC SAMPLING}

\begin{abstract}
The present article tries about the statistical methodology implemented for the supervision of the installation for phone lines, by means of the calculation of an established parameter inside the supervision procedures that measures the grade of execution of the service offered by the concessionary company.
\end{abstract}

KEYWORDS.- Probabilistic sampling. Mensuration parameters of Supervision

\section{INTRODUCCIÓN}

Telefónica del Perú S.A. es actualmente el principal concesionario de las telecomunicaciones que opera en el Perú y está habilitado para explotar comercialmente determinados servicios de telecomunicaciones, según el contrato de Concesión firmado con el Estado Peruano.

Por otro lado está OSIPTEL el organismo supervisor de la inversión privada en Telecomunicaciones, quien tiene como una de sus principales funciones controlar la calidad de servicio que Telefónica brinda a sus usuarios.

Son muchos los problemas presentados a OSIPTEL en su función de organismo supervisor, la mayoría de estos problemas requieren inspecciones basadas en muestras, ya sea de usuarios, teléfonos públicos, celulares, solicitudes de instalación, recibos telefónicos, etc.

Veamos a continuación algunos de estos problemas:

- Modificación del Sistema Tarifario

Se cambió el sistema de tarifa, para medir la duración de la llamada telefónica en pasos de un minuto, en vez de tres minutos como funcionaba antes.

En la solución de este problema se buscó el mejor modelo de probabilidad para la duración de llamadas telefónicas. Utilizando el programa BESTFIT se encontró un modelo como el que se muestra en la figura 1.

\footnotetext{
' Universidad Nacional Mayor de San Marcos. Facultad de Ciencias Matemáticas. e-mail: jramosr@unmam.edu.pe, npillhuamanc@unmsm.edu.pe
} 


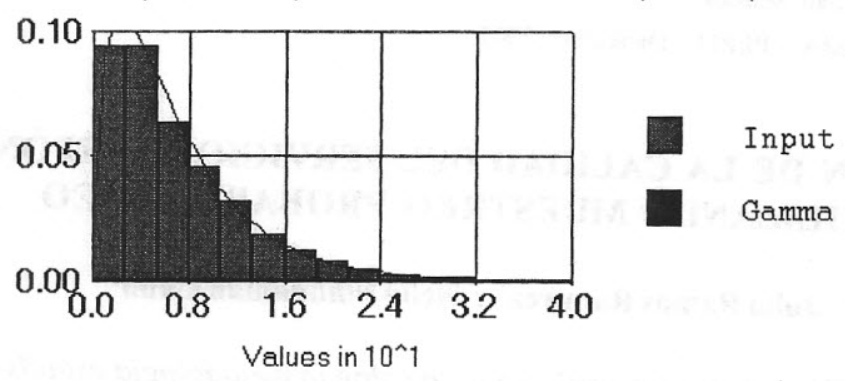

- Devoluciones monetarias a los usuarios de telefonía fija y móvil, por cobros indebidos.

- Calidad de servicio en la Red de telefonía móvil, mediante la estimación estadística de parámetros de calidad (indicadores):

- Grado de Servicio

- Calidad de la comunicación

- Cobertura radioeléctrica.

- Calidad de servicio de los Teléfonos Públicos Instalados (TPI), mediante la inspección en campo de una muestra de TPI.

- Tráfico telefónico en Centros Poblados Rurales (CPR), mediante la medición de demanda de servicios de telecomunicación.

- Atención de solicitudes de instalación de líneas telefónicas en tiempos establecidos.

\section{EL PROBLEMA DE INVESTIGACIÓN}

Una de las funciones del OSIPTEL es la supervisión de la instalación de líneas telefónicas en un tiempo establecido en los contratos de concesión. Un parámetro establecido dentro de los procedimientos de supervisión, que mide el grado de este cumplimiento es denotado por $\theta$.

El parámetro $\theta$ se define como

$$
\theta=\frac{N_{1}}{N-N_{2}}
$$

donde:

$\mathrm{N}$ = Número total de solicitudes presentadas

$\mathrm{N}_{1}=$ Número de solicitudes atendidas

$\mathrm{N}_{2}=$ Número de solicitudes excluidas

$\mathrm{N}_{3}=$ Número de solicitudes no atendidas

Por lo tanto $N=\sum_{i=1}^{3} N_{i}$

Por otro lado, si dividimos por $\mathrm{N}$ en el numerador y denominador, el parámetro se puede expresar como

$$
\theta=\frac{\pi}{1-p}
$$

donde 
$\pi=\frac{N_{1}}{N}=$ Proporción de solicitudes atendidas sobre el total de solicitudes presentadas.

$p=\frac{N_{2}}{N}=$ Proporción de solicitudes excluidas sobre el total de solicitudes presentadas.

Este parámetro entró en vigencia a partir de 1998.

El problema de investigación desde el punto de vista estadístico es

- ¿Cómo obtener una estimación con alta precisión del parámetro $\theta$ ?

- ¿Cuál es el diseño óptimo de muestreo, que permita una estimación adecuada del parámetro $\theta$ ?

\section{DISEÑO MUESTRAL}

Veamos a continuación los componentes del diseño muestral.

\subsection{Población Objetivo}

Está formado por el conjunto de todas las solicitudes de instalación de teléfonos presentadas y reportadas por el concesionario en Lima y Provincias.

La población se ha dividido en dos dominios de estudio.

El dominio Lima: comprende todas las solicitudes presentadas en los 10 departamentos de mantenimiento de Lima.

El dominio Provincias: comprende todas las solicitudes presentadas independientemente en cada una de las 11 Oficinas Zonales de Provincias.

Los dominios de estudio de Lima y Provincias se pueden observar en el cuadro de tamaños de muestra presentado en el Cuadro No.1 del Anexo.

\subsection{Marco de Muestreo}

Se dispone, de un marco de muestreo para cada dominio de estudio. El marco, para el dominio Lima, está conformado por el listado de solicitudes presentadas y reportadas por el concesionario que abarca los 10 departamentos de mantenimiento de Lima. Para el dominio Provincias, se dispone de un marco independiente por cada Oficina Zonal.

Con el propósito de no introducir errores ajenos al muestreo, el personal de campo realizó un proceso de depuración del Marco de Muestreo, antes del proceso de selección de la muestra.

\subsection{Plan de muestreo}

En cada dominio de estudio se seleccionará independientemente una muestra de solicitudes presentadas a partir del Marco de Muestreo depurado.

\section{Dominio Lima}

La selección de la muestra en este dominio se realizará según el Muestreo Probabilístico Sistemático.

A continuación se describe el procedimiento de selección del Muestreo Probabilístico Sistemático. 
Sea: $\mathrm{N}=$ Número de solicitudes presentadas en el Marco de Muestreo.

$\mathrm{n}=$ Número de solicitudes presentadas que serán seleccionadas.

$\mathrm{K}=\mathrm{N} / \mathrm{n}=$ Intervalo de selección constante. ( $\mathrm{K}$ debe ser redondeado al máximo entero)

Por ejemplo, si $\mathrm{K}=31.3$ debe ser redondeado a $\mathrm{K}=31$ si $\mathrm{K}=31.8$ debe ser redondeado a $\mathrm{K}=31$

El procedimiento de selección consiste

1ro. Seleccionar un número aleatorio (arranque aleatorio) entre 1 y K. Denotemos con $\mathbf{r}$ al arranque aleatorio.

2do. La muestra quedará formada por las solicitudes presentadas que ocupan los lugares:

$$
r, r+K, r+2 K, \ldots \ldots, r+(n-1) K
$$

\section{Dominio Provincias}

La selección de la muestra en este dominio se realizará según el Muestreo Estratificado, con selección sistemática en cada estrato independientemente. Un estrato es equivalente a una oficina zonal y estará conformado por todas las solicitudes presentadas por el concesionario en dicha oficina zonal.

\subsection{Tamaño de muestra para el dominio Lima}

\section{Fórmula Propuesta para el Tamaño de Muestra}

Se calculará un tamaño de muestra independientemente para cada Dominio de Estudio. La fórmula utilizada para el cálculo del tamaño de la muestra en el dominio Lima, ha sido desarrollada según el Plan de Muestreo presentado en 3.3 y la información disponible para su aplicación.

Entonces el número de solicitudes presentadas que serán seleccionadas en el dominio Lima, se calculará con la fórmula

$$
n=\frac{N\left[\theta(1-\theta)+\theta^{2} P\right]}{(N-1)(1-P) D+\left[\theta(1-\theta)+\theta^{2} P\right]}
$$

donde:

$\mathrm{N}=$ Número total de solicitudes presentadas en el dominio Lima

$\theta=$ Valor del indicador $\theta$ reportado por TdP en el dominio Lima

$\mathrm{P}=$ Proporción de solicitudes excluidas sobre el total de solicitudes presentadas por $\mathrm{TdP}$ en el dominio Lima.

$$
D=\left(\frac{E}{1.96}\right)^{2}
$$

$\mathrm{E}=0.05=$ Máximo error de estimación que se espera cometer, con una confianza del $95 \%$.

\section{Cálculo de los Tamaños de Muestra}

El tamaño de Muestra con su respectiva fracción de muestreo para el dominio Lima, fue calculado bajo el método propuesto según la fórmula anterior. Los resultados se presentan en el cuadro 1 del Anexo. En dicho Anexo también se presenta el tamaño de muestra para el dominio Lima bajo 
el método convencional, el cual no contempla la información respecto al número de solicitudes excluidas por el concesionario.

La fórmula que se utiliza bajo el método convencional está dado por

$$
n=\frac{N[\theta(1-\theta)]}{(N-1) D+[\theta(1-\theta)]}
$$

Se puede observar que la proporción de solicitudes excluidas por el concesionario (parámetro P) no es parte de esta fórmula, a diferencia de la primera fórmula que si incluye este dato. Lo más importante se da en el hecho que la primera fórmula coincide con el método convencional cuando $\mathrm{P}=0$, es decir cuando ninguna solicitud haya sido excluida (una situación irreal).

Se observa también que conforme se incrementa el valor de $\mathrm{P}$, el tamaño de muestra propuesto también se incrementa (ver cuadro 1 del Anexo).

\subsection{Tamaño de muestra en el dominio provincia}

\section{Fórmula Propuesta para el Tamaño de Muestra}

Se calculará un tamaño de muestra global para todos los estratos y luego se distribuirá proporcionalmente a cada estrato. La fórmula utilizada para el cálculo del tamaño de la muestra en el dominio Provincia, ha sido desarrollada según el Plan de Muestreo presentado en 3.3 y la información disponible para su aplicación.

Entonces el número de solicitudes presentadas que serán seleccionadas en el dominio Provincia, se calculará con la fórmula

$$
n=\frac{\sum_{i=1}^{L} \frac{W_{i} \pi_{i}\left(1-\pi_{i}\right)}{\left(1-P_{i}\right)^{2}}}{D\left(1-\sum_{i=1}^{L} W_{i} P_{i}\right)^{2}}
$$

donde:

$W_{i}=$ Ponderación del $\mathrm{i}$ - ésimo estrato (oficina zonal)

$\pi_{i}=\theta_{i}\left(1-P_{i}\right)$

$P_{i}=$ Proporción de solicitudes excluidas sobre el total de solicitudes presentadas por

$\mathrm{TdP}$ en el i - ésimo estrato.

$\theta_{i}=$ Valor del indicador $\theta$ en el $\mathrm{i}$ - ésimo estrato.

$L=$ Número de estratos.

$E=0.05=$ Máximo error de estimación que se espera cometer, con una confianza del $95 \%$.

Luego de calcularse el tamaño de muestra global para todos los estratos, se distribuye proporcionalmente a cada estrato según la fórmula:

$$
n_{i}=n W_{i}
$$




\section{Cálculo de los Tamaños de Muestra}

Los tamaños de muestra con sus respectivas fracciones de muestreo para cada estrato del dominio provincia, fueron calculados bajo el método propuesto según la fórmula anterior.

Los resultados se presentan en el cuadro 1 del Anexo. En este Anexo también se presenta el tamaño de muestra para el dominio Provincia bajo el método convencional, el cual no contempla la información respecto al número de solicitudes.

\section{Distribución del Tamaño de Muestra en Oficinas Zonales de Provincias}

Las solicitudes presentadas reportadas por el concesionario para una oficina zonal en provincias, corresponde a varias localidades de la zona. La muestra de una oficina zonal se distribuirá proporcionalmente al número de solicitudes atendidas por localidad.

Definamos lo siguiente:

$L=$ Número de localidades de la oficina zonal.

$N_{i}=$ Número total de solicitudes atendidas en la i -ésima localidad de la oficina zonal.

$N=\sum_{i=1}^{L} N_{i}$ Número total de solicitudes atendidas en la oficina zonal.

$n$ = Número de solicitudes presentadas que serán seleccionadas en la oficina zonal.

$n_{i}=$ Número de solicitudes presentadas que serán seleccionadas en la oficina zonal.

Luego $n_{i}$ se calcula con la fórmula

$$
n_{i}=n\left(\frac{N_{i}}{N}\right)
$$

\section{PROCESO DE ESTIMACIÓN}

\section{Parámetros a estimar}

En un proceso de estimación basado en una muestra, usualmente se desea hacer una estimación puntual del parámetro de interés, en este caso el interés es por el parámetro $\theta$. Sin embargo, cuando se propone hacer alguna inferencia o una generalización (expansión) de los resultados muestrales hacia la población de estudio, es necesario estimar también otros parámetros que permitan tal propósito. Estos parámetros adicionales son: varianza del estimador, error estándar del estimador, error de estimación (margen de error) y el error de muestreo con un cierto nivel de confiabilidad. Estas estimaciones nos conducen a realizar una estimación interválica que nos permitirán realizar una inferencia adecuada hacia la población.

\section{Estimador del parámetro $\theta$}

Como se puede apreciar el parámetro $\theta$ depende de otros dos parámetros, $\pi$ y $P$, y ambos son desconocidos por OSIPTEL. Por lo tanto, al estimar ambos parámetros se obtendrá por consecuencia el estimador del parámetro $\theta$. Tanto $\pi$ y $P$ son parámetros de proporción y sus estimadores máximo verosímiles son respectivamente:

$$
\hat{\pi}=\frac{X}{n} \quad \hat{P}=\frac{Y}{n}
$$


Donde:

$X=$ número de solicitudes atendidas encontradas en la muestra

$Y=$ número de solicitudes excluidas encontradas en la muestra

$n=$ número de solicitudes en la muestra

Luego el estimador puntual del parámetro $\theta$ es dado por

$$
\hat{\theta}=\frac{\hat{\pi}}{1-\hat{P}}=\frac{X}{n-Y}
$$

\section{Estimador de la Varianza de $\hat{\theta}$}

Según la teoría estadística, $X$ e $Y$ son variables aleatorias con distribución de probabilidad Binomial y $\hat{\theta}$ es una variable aleatoria que tiene la forma de un estimador de razón. Los estimadores de razón son estimadores no lineales sesgados, pero son bastante prácticos y útiles en la estimación de parámetros.

Cuando un estimador es sesgado la precisión y estabilidad del estimador se mide mediante el error cuadrático medio. En el caso del estimador de razón existe una aproximación basada en el desarrollo de Series de Taylor para la varianza y el estimador de varianza. En base a este desarrollo se establece

la fórmula del estimador de varianza de $\hat{\theta}$. La fórmula es la siguiente:

$$
v(\hat{\theta})=\left(\frac{1}{n}-\frac{1}{N}\right)\left(\frac{1}{1-\hat{P}}\right)^{2}\left[\frac{n \hat{\pi}(1-\hat{\pi})}{n-1}-\frac{2 n \hat{\pi}^{2} \hat{P}}{(n-1)(1-\hat{P})}+\frac{n \hat{\pi}^{2} \hat{P}}{(n-1)(1-\hat{P})}\right]
$$

La fórmula anterior de varianza es importante porque mide la precisión del estimador del parámetro $\theta$, determinando la estabilidad o inestabilidad de dicho estimador.

\section{Error Estándar de $\hat{\theta}$}

Para una real interpretación de la precisión del estimador es necesario medirlo y expresarlo en las mismas unidades de la variable original, pues la varianza se expresa en unidades cuadráticas. En ese sentido la desviación estándar es una medida de la precisión del estimador expresado en las mismas unidades de la variable original y se define como

$$
E E=\sqrt{v(\hat{\theta})}
$$

\section{Estimación interválica}

Para lograr una estimación interválica del parámetro $\theta$ se tiene que evaluar previamente algunos elementos estadísticos, tales como: error máximo de estimación, nivel de confianza, límite inferior y superior de confianza. Definamos a continuación estos términos.

\section{Error máximo de estimación}

La teoría de Normalidad Asintótica permite que el estimador $\hat{\theta}$ se distribuya aproximadamente como un modelo Normal y por lo tanto se cumple

$$
P(|\hat{\theta}-\theta|<E)=\gamma
$$


Donde:

$$
\begin{aligned}
& E=1.96=\sqrt{v(\hat{\theta})}=\text { error máximo de estimación (margen de error) } \\
& \gamma=0.95=\text { nivel de confianza (probabilidad) }
\end{aligned}
$$

El error máximo de estimación es el límite máximo que alcanza el verdadero error de estimación absoluto y es calculado con una confiabilidad del $95 \%$.

\section{Límites de confianza}

Los límites de confianza, superior e inferior, son consecuencia de la estimación puntual y del error máximo de estimación. Los límites son:

Límite Inferior de Confianza (LIC) $=\theta-E$

Límite Superior de Confianza (LSC) $=\theta-E$

\section{Intervalo de confianza}

Finalmente obtenemos el intervalo del $95 \%$ de confianza para el parámetro $\theta$ dado por $(\hat{\theta}-E, \hat{\theta}+E)$. Este intervalo contiene al parámetro $\theta$ con una seguridad del $95 \%$. Es decir, en el $95 \%$ de todas las muestras posibles se obtendrá intervalos de confianza que contienen al parámetro $\theta$.

\section{RESULTADOS Y EVALUACIÓN DE LA ESTIMACIÓN DEL PARÁMETRO $\theta$}

\section{Resultados de la estimación del parámetro}

Los resultados que se presentan en los cuadros 2 y 3 del Anexo, se refieren a la estimación puntual e interválica del parámetro $\theta$, para el dominio Lima y para el dominio Provincias.

En el cuadro No.2 se presentan los resultados considerando como solicitudes excluidas sólo aquellas solicitudes de la muestra que tienen algún sustento para la exclusión (es decir, aquellas que realmente debieron ser excluidas por el concesionario). Las solicitudes que fueron excluidas sin sustento fueron consideradas como solicitudes no atendidas.

En el cuadro No.3 se presentan los resultados considerando como solicitudes excluidas aquellas solicitudes de la muestra que tienen o no sustento para la exclusión (es decir, todas aquellas que el concesionario reportó como excluidas). Entonces las solicitudes que fueron excluidas sin sustento fueron consideradas como solicitudes excluidas.

Comparando los resultados de ambos cuadros ( 2 y 3), es evidente que el cuadro No.3 favorece ampliamente al concesionario, ya que se está sobrestimando el parámetro $\theta$ alrededor de 10 puntos porcentuales adicionales. Esto es consecuencia de haber considerado como solicitudes excluidas aquellas que el concesionario excluyó sin sustento. Por otro lado, es importante señalar que el propósito de la investigación es obtener una estimación de alta precisión del parámetro $\theta$ efectuando para ello inspecciones rigurosas que conlleven a dicho propósito. Por tanto, los resultados a evaluar y analizar por OSIPTEL se centran en el cuadro No.2.

\section{Evaluación de la estimación del parámetro $\theta$}

Para evaluar la bondad de las estimaciones logradas para el parámetro $\theta$ se debe obtener y analizar el error máximo de estimación, el error de muestreo, los límites de confianza y el nivel de confianza utilizado. Esta evaluación se realiza a continuación tanto para el dominio Lima como para el dominio Provincias, basados en el cuadro No.2. 


\section{Dominio Lima}

Con estos resultados muestrales se estimó el parámetro $\theta$ en $83.56 \%$ con un error máximo de estimación de más o menos $2.42 \%$. Esto implica que el parámetro $\theta$ está entre el $81.13 \%$ y $85.98 \%$. Como el nivel de confianza utilizado fue de $95 \%$, entonces podemos afirmar que estos resultados son válidos en el $95 \%$ de todas las muestras posibles y solamente en el $5 \%$ de los casos tendremos resultados muy diferentes a los anteriores.

Por otro lado recordemos que el diseño de la muestra para la estimación del parámetro $\theta$ contempló un error máximo de estimación del $5 \%$ dispuesto a tolerar, y sin embargo se ha obtenido sólo $2.42 \%$ que es mucho menor. Esto es un buen indicador de la precisión y la bondad de la estimación del parámetro $\theta$.

Otro indicador de la precisión de la estimación del parámetro $\theta$ es el error de muestreo que es el porcentaje máximo de error respecto a la estimación; es decir, $\frac{2.42}{83.56} \times 100=2.90 \%$ que es un porcentaje bastante pequeño si se tiene en cuenta que en el muestreo se tolera usualmente hasta $5 \%$ y en algunos casos $10 \%$.

\section{Dominio Provincias}

En el dominio Provincias se tiene un total de 76272 solicitudes presentadas en las diferentes oficinas departamentales tal como se muestra en el cuadro No.1, de las cuales se seleccionó una muestra total de 4627 solicitudes seleccionadas mediante el plan de muestreo descrito en el informe "Diseño de muestra para la estimación del parámetro $\theta$ ". En la muestra se encontró en total 3557 solicitudes atendidas, 354 no atendidas y 716 excluidas ( 385 con sustento y 331 sin sustento).

Con estos resultados muestrales se estimó el parámetro $\theta$ a nivel de provincias en $84.39 \%$ con un error máximo de estimación de más o menos $1.04 \%$. Esto implica que el parámetro $\theta$ en provincias está entre el $83.35 \%$ y $85.44 \%$. Como el nivel de confianza utilizado fue de $95 \%$, entonces podemos afirmar que estos resultados son válidos en el $95 \%$ de todas las muestras posibles y solamente en el $5 \%$ de los casos tendremos resultados muy diferentes a los anteriores.

Por otro lado recordemos que el diseño de la muestra para la estimación del parámetro $\theta$ en provincias contempló también un error máximo de estimación de $15 \%$ dispuesto a tolerar, y sin embargo se ha obtenido sólo $1.04 \%$ que es bastante pequeño. Esto es un buen indicador de la precisión y la bondad de la estimación del parámetro $\theta$ en provincias.

Adicionalmente, el error de muestreo para el parámetro $\theta$ en provincias es que es un porcentaje $\frac{1.04}{84.39} \times 100=1.23 \%$ muy pequeño y bastante favorable para el proceso de estimación.

\section{CONCLUSIONES}

Las principales conclusiones son las siguientes:

- El método propuesto para el cálculo del tamaño de muestra es mejor que el método convencional. Ambos métodos coinciden cuando el parámetro $P=0$, lo que significaría la no existencia de solicitudes excluidas, algo que no es real.

El método propuesto para el dominio Lima permitirá obtener una estimación de alta precisión para el parámetro $\theta$ a nivel del dominio, más no a nivel de departamento de mantenimiento. El método propuesto para el dominio Provincia permitirá obtener una estimación de alta precisión a nivel de dominio y a nivel de estratos.

- Se logró una estimación de alta precisión para el parámetro TEC en ambos dominios (Lima y Provincias), ya que los niveles de error reportados son bastante pequeños. 
- La alta precisión lograda se debe a un diseño óptimo de muestreo, fundamentalmente a un buen manejo del tamaño de la muestra, al proceso de selección de la muestra y a las inspecciones rigurosas en el trabajo de campo realizado por OSIPTEL.

- Según los resultados muestrales, en el dominio Lima, el $70.71 \%$ de solicitudes excluidas por el concesionario no tienen sustento; mientras que en el dominio Provincias, el $46.23 \%$ de solicitudes excluidas por el concesionario no tienen sustento. En ambos dominios las exclusiones sin sustento son bastante altas y OSIPTEL debería prestar bastante atención a este hecho. Esto nos ha llevado a dos resultados. El cuadro No.1 nos estaría proporcionando una estimación cercana a la realidad y el cuadro No.2 nos proporciona una sobrestimación del parámetro $\theta$ favorable al concesionario. Por tanto, se recomienda que para el análisis y decisiones que OSIPTEL requiera implantar, se utilice los resultados dados en el cuadro No.2.

- Con los resultados obtenidos en el dominio Lima en base a los resultados del cuadro No.2, específicamente en el intervalo de confianza que está entre $81.13 \%$ y $85.98 \%$, podemos inferir que "si hay evidencia estadística suficiente para afirmar que el concesionario está cumpliendo con el 80\% mínimo establecido en los contratos de concesión".

- En el dominio Provincias, "si hay evidencia estadística suficiente para afirmar que el concesionario está cumpliendo con el 80\% mínimo establecido en los contratos de concesión”. Sin embargo, hay zonas departamentales como Tacna y Piura cuyos niveles están muy por debajo del $80 \%$ mínimo establecido.

\section{Cuadro No 1: Tamaños de muestra en los dominios Lima y Provincias}

\begin{tabular}{|c|c|c|c|c|c|c|c|c|c|}
\hline & & & & & & & TAMAÑO DE & MUESTF & (n) \\
\hline & & & & & & METOD & PROPUESTO & METODC & ONVENCIONAL \\
\hline \begin{tabular}{|c|} 
DPTOS. DE \\
MANTENIMIEN \\
TO \\
LIMA \\
\end{tabular} & $\begin{array}{c}\text { PRESENTADAS } \\
\text { REPORTE } \\
\text { ANUAL-TdP } \\
\end{array}$ & $\begin{array}{l}\text { ATENDIDAS } \\
\text { REPORTE } \\
\text { ANUAL-TdP }\end{array}$ & \begin{tabular}{|c|} 
EXCLUIDAS \\
REPORTE \\
ANUAL-TdP
\end{tabular} & $\theta$ & $\mathbf{P}$ & $n$ & $\begin{array}{l}\text { Fracción } \\
\text { Muestreo }\end{array}$ & $\mathbf{n}$ & \begin{tabular}{|l|} 
Fracción \\
Muestreo
\end{tabular} \\
\hline Santa Patricia & 23983 & 16095 & 6868 & 0.9404032 & 0.2863695 & 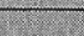 & & & \\
\hline \begin{tabular}{|l|} 
Miraflores \\
\end{tabular} & 15355 & 9710 & 4676 & 0.9092612 & 0.3045262 & & & & \\
\hline Chorrillos & 25360 & 15136 & 9159 & 0.9342633 & 0.3611593 & 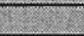 & {$[2$} & & \\
\hline Higuereta & 14109 & 8340 & 5282 & 0.9448284 & 0.374371 & $\overline{2}$ & & & \\
\hline La Victoria & 27637 & 15822 & 11342 & 0.9709727 & 0.4103919 & $=$ & 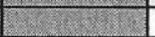 & & \\
\hline San Jose & 16827 & 11374 & 5067 & 0.9671769 & 0.3011232 & - & & & \\
\hline \begin{tabular}{|l|} 
Magdalena \\
\end{tabular} & 15677 & 10198 & 5050 & 0.9596311 & 0.322128 & - & - & & \\
\hline $\begin{array}{l}\text { Los Olivos } \\
\end{array}$ & 28251 & 16150 & 10280 & 0.8986701 & 0.3638809 & & & & \\
\hline Washington & 17825 & 10458 & 6885 & 0.9559415 & 0.3862553 & & & & \\
\hline Lima Provincia & 1990 & 627 & 1340 & 0.9646154 & 0.6733668 & & & & \\
\hline TOTAL & 139374 & 113910 & 18309 & 0.9408995 & 0.131366 & 303 & $0.22 \%$ & 85 & $0.06 \%$ \\
\hline
\end{tabular}

\begin{tabular}{|c|c|c|c|c|c|c|c|c|c|}
\hline $\begin{array}{c}\text { OFICINAS } \\
\text { ZONALES } \\
\text { PROVINCIAS }\end{array}$ & $\begin{array}{l}\text { PRESENTADAS } \\
\text { REPORTE } \\
\text { ANUAL-TdP }\end{array}$ & $\begin{array}{l}\text { ATENDIDAS } \\
\text { REPORTE } \\
\text { ANUAL-TdP }\end{array}$ & $\begin{array}{l}\text { EXCLUIDAS } \\
\text { REPORTE } \\
\text { ANUAL-TdP }\end{array}$ & $\theta$ & $P$ & n & $\begin{array}{l}\text { Fracción de } \\
\text { Muestreo }\end{array}$ & n & $\mid \begin{array}{ll}\text { Fracción } & \text { de } \\
\text { Muestreo } & \end{array}$ \\
\hline Arequipa & 9648 & 6380 & 1740 & 0.8067779 & 0.1803483 & 791 & $8.20 \%$ & 53 & $0.55 \%$ \\
\hline Chiclayo & 9307 & 7127 & 1714 & 0.9386277 & 0.1841625 & 763 & $8.20 \%$ & 51 & $0.55 \%$ \\
\hline Chimbote & 5935 & 5032 & 497 & 0.9253402 & 0.0837405 & 487 & $8.20 \%$ & 32 & $0.55 \%$ \\
\hline Cusco & 5228 & 4044 & 619 & 0.8774138 & 0.1184009 & 429 & $8.20 \%$ & 29 & $0.55 \%$ \\
\hline Huancayo & 9622 & 7545 & 1367 & 0.9139915 & 0.1420703 & 789 & $8.20 \%$ & 53 & $0.55 \%$ \\
\hline Ica & 3884 & 3114 & 582 & 0.9430648 & 0.1498455 & 319 & $8.20 \%$ & 21 & $0.55 \%$ \\
\hline Iquitos & 8491 & 7091 & 1031 & 0.9505362 & 0.1214227 & 696 & $8.20 \%$ & 46 & $0.55 \%$ \\
\hline Piura & 7449 & 5400 & 1381 & 0.8899143 & 0.185394 & 611 & $8.20 \%$ & 41 & $0.55 \%$ \\
\hline Tacna & 3597 & 2274 & 1001 & 0.875963 & 0.2782875 & 295 & $8.20 \%$ & 20 & $0.55 \%$ \\
\hline Trujillo & 13111 & 8814 & 3226 & 0.891654 & 0.2460529 & 1075 & $8.20 \%$ & 72 & $0.55 \%$ \\
\hline \begin{tabular}{|l|} 
Lima Resto \\
\end{tabular} & 2465 & 1580 & 325 & 0.7383178 & 0.1318458 & 202 & $8.20 \%$ & 13 & $0.55 \%$ \\
\hline TOTAL & 78737 & 58401 & 13483 & & & 6458 & $8.20 \%$ & 431 & $0.55 \%$ \\
\hline
\end{tabular}


Cuadro No.2. Estimación del Indicador TEC - Se consideró como EXCLUIDAS sólo las solicitudes que tienen sustento

\begin{tabular}{|c|c|c|c|c|c|c|c|c|c|c|c|c|c|}
\hline OFICINA & 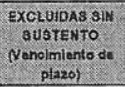 & $\begin{array}{c}\text { EXCLUIDAB } \\
\text { CON } \\
\text { BUSTENTO }\end{array}$ & $\begin{array}{c}\text { TOTAL } \\
\text { Excluidas }\end{array}$ & $\begin{array}{c}\text { No } \\
\text { ATENDIDAS }\end{array}$ & ATEMDIDAS & \begin{tabular}{|} 
TOTAL \\
MUEOTRA
\end{tabular} & $\begin{array}{l}\text { TOTAL UNVREO } \\
\text { SOLIC. } \\
\text { PRESENTABAS }\end{array}$ & TEC & $\pi$ & P & Var & ERROR & $\begin{array}{c}\text { LDMITE } \\
\text { INFERIOR DE } \\
\text { ESTIMACION }\end{array}$ \\
\hline 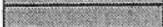 & & & & & & & & & & & & & \\
\hline LiMA+(lima-prov) & 99 & 41 & 140 & 48 & 747 & 935 & 139374 & $83.56 \%$ & 0.7989 & 0.0439 & 0.0001528 & $2.42 \%$ & $81.13 \%$ \\
\hline cusco & 9 & 37 & 46 & 43 & 311 & 400 & 5228 & $85.67 \%$ & 0.7775 & 0.0925 & 0.000313 & $3.47 \%$ & $82.21 \%$ \\
\hline AREQUIPA & 47 & 57 & 104 & 70 & 442 & 616 & 9648 & $79.07 \%$ & 0.7175 & 0.0925 & 0.0002776 & $3.27 \%$ & $75.80 \%$ \\
\hline ICA & 11 & 11 & 22 & 19 & 242 & 283 & 3884 & $88.97 \%$ & 0.8551 & 0.0389 & 0.0003357 & $3.59 \%$ & $85.38 \%$ \\
\hline TACNA & 36 & 33 & 69 & 23 & 131 & 223 & 3597 & $68.95 \%$ & 0.5874 & 0.1480 & 0.0010617 & 6.39\% & $62.56 \%$ \\
\hline HEANCAYO & 25 & 54 & 79 & 47 & 440 & 566 & 9622 & $85.94 \%$ & 0.7774 & 0.0954 & 0.0002225 & $2.92 \%$ & $83.01 \%$ \\
\hline CHIMBOTE & 10 & 8 & 18 & 10 & 192 & 220 & 5935 & $90.57 \%$ & 0.8727 & 0.0364 & 0.0003899 & $3.87 \%$ & $86.70 \%$ \\
\hline TRUJILLO & 86 & 35 & 121 & 38 & 576 & 735 & 13111 & $82.29 \%$ & 0.7837 & 0.0476 & 0.0001968 & $2.75 \%$ & $79.54 \%$ \\
\hline CHICLAYYO & 16 & 106 & 122 & 23 & 518 & 663 & 9307 & $93.00 \%$ & 0.7813 & 0.1599 & 0.0001087 & $2.04 \%$ & $90.95 \%$ \\
\hline PIURA & 84 & 28 & 112 & 60 & 389 & 561 & 7449 & $72.98 \%$ & 0.6934 & 0.0499 & 0.0003427 & $3.63 \%$ & $69.35 \%$ \\
\hline वियाTos & 7 & 16 & 23 & 21 & 316 & 36 & 8491 & $91.86 \%$ & 0.8778 & 0.0444 & 0.0002087 & $2.83 \%$ & $89.03 \%$ \\
\hline TOTAL & 331 & 385 & 716 & 354 & 3557 & 4627 & 76272 & $84.39 \%$ & 0.7687 & 0.0832 & $2.834 \mathrm{E}-05$ & $1.04 \%$ & $83.35 \%$ \\
\hline
\end{tabular}

Cuadro No.3. Estimación del Indicador TEC - Se consideró como EXCLUIDAS las solicitudes que tienen sustento y sin sustento

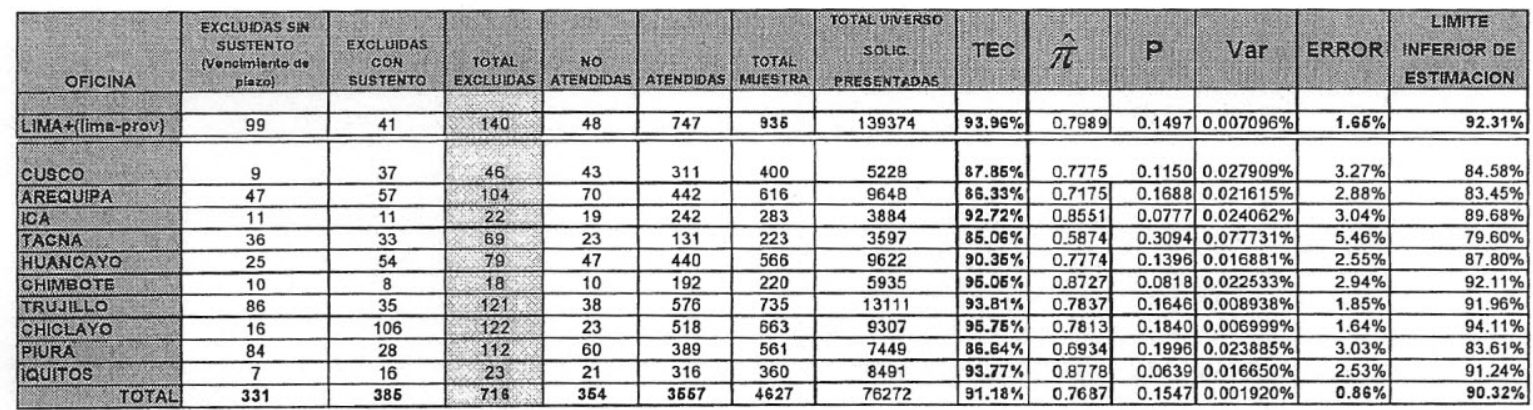

